\title{
Spatially Resolved Investigation of Solution Cast Nanoparticle Films by X-ray Scattering and Multidimensional Data Set Classification
}

\author{
Stephan V. Roth, ${ }^{*}{ }^{\dagger}$ André Rothkirch,${ }^{\dagger}$ Tina Autenrieth, ${ }^{\dagger}$ Rainer Gehrke, ${ }^{\dagger}$ Thomas Wroblewski, ${ }^{\dagger}$ \\ Manfred C. Burghammer, ${ }^{\star}$ Christian Riekel, ${ }^{\sharp}$ Leander Schulz, ${ }^{\S}$ Rosmarie Hengstler, ${ }^{\|, \perp}$ and \\ Peter Müller-Buschbaum" \\ ${ }^{\dagger}$ Deutsches Elektronen-Synchrotron (DESY), Notkestr. 85, D-22607 Hamburg, Germany, ${ }^{\star}$ European \\ Synchrotron Radiation Facility (ESRF), 6 rue Jules Horowitz, F-38043 Grenoble, France, ${ }^{\S}$ Physics Department, \\ Université de Fribourg, Chemin du Musée 3, CH-1700 Fribourg, Switzerland, and I" Physik-Department E13, \\ Technische Universität München, James-Franck-Str. 1, D-85748 Garching, Germany. ${ }^{\perp}$ Current address:
} AREVA NP GmbH, Paul-Gossen-Str. 100, 91052 Erlangen, Germany

\begin{abstract}
A combinatorial high-throughput approach is used to investigate a solution cast gradient consisting of colloidal gold nanoparticles on top of a silicon substrate by means of a X-ray nanobeam. Classification algorithms are used to reveal and visualize structural transitions from a frozen colloidal solution to a well-defined nanostructure. Prominent length scales on the order of $100 \mathrm{~nm}$ are observed. A periodic change in the nanostructure along the gradient is explained by a simplified stick-slip model.
\end{abstract}

The fundamental understanding of wetting and flow behavior of nanoparticle and (bio)polymeric solutions and blends on solid substrates is crucial for their application in many technological fields, for example, proteomics, optical coatings, or data storage applications. $^{1-6}$ Typical deposition methods are spin-coating, ${ }^{7}$ Langmuir-Blodgett/Langmuir-Schaefer ${ }^{8}$ techniques, or solution casting. ${ }^{9,10}$ The latter is widely applied in producing designed colloidal, polymeric, and biopolymeric thin films. ${ }^{41,12}$ It allows for realizing dedicated morphologies and morphological gradients in order to perform combinatorial investigations of thin films ${ }^{4,9}$ for different applications, for example, to produce two- and threedimensional photonic crystals. ${ }^{13,14}$ Combinatorial methods ${ }^{15,16}$ are routinely used for material testing and optimization. By testing a large physical parameter space in short time, one is able to determine the structure-function relationship desired from the

*To whom correspondence should be addressed. E-mail: stephan.roth@, desy.de.

(1) Erokhin, V.; Troitsky, V.; Erokhina, S.; Mascetti, G.; Nicolini, C. Langmuir 2002, 18,3185

(2) Bauer, G.; Hassmann, J.; Walter, H.; Haglmüller, J.; Mayer, C.; Schalkhammer, T. Nanotechnology 2003, 14, 1289.

(3) Walter, H.; Bauer, G.; Domnick, R.; Jakopic, G.; Leitner, A. Opt. Eng. 2006, 45,103801 .

(4) Gebhardt, R.; Vendrely, C.; Burghammer, M.; Riekel, C. Langmuir 2009, 25, 6307.

(5) Nicolini, C.; Pechkova, E. J. Nanosci. Nanotechnol. 2006, 6, 2209.

(6) Abul Kashem, M. M.; Perlich, J.; Schulz, L.; Roth, S. V.; Müller-Buschbaum,

P. Macromolecules 2008, $41,2186$.

(7) Müller-Buschbaum, P. Anal. Bioanal. Chem. 2003, 376, 3 .

(8) Pechkova, E.; Innocenzi, P.; Malfatti, L.; Kidchob, T.; Gaspa, L.; Nicolini, C. Langmuir 2007, 23, 1147.

(9) Gebhardt, R.; Burghammer, M.; Riekel, C.; Roth, S. V.; Müller-Buschbaum, P. Macromol. Biosci. 2008, 8, 347.

(10) Kuhlmann, M.; Feldkamp, J. M.; Patommel, J.; Roth, S. V.; Timmann, A.; Gehrke, R.; Müller-Buschbaum, P.; Schroer, C. G. Langmuir 2009, $25,7241$.

(11) Roth, S. V.; Autenrieth, T.; Grübel, G.; Riekel, C.; Burghammer, M.; Hengstler, R.; Schulz, L.; Müller-Buschbaum, P. Appl. Phys. Lett. 2007, 91, 091915.

(12) Lechmann, M. C.; Kessler, D.; Gutmann, J. S. Langmuir 2009, 25, 10202.

(13) Xia, Y.; Gates, B.; Yin, Y.; Lu, Y. Adv. Mater. 2000, 12, 693.

(14) Hu, S.; Men, Y.; Roth, S.; Gehrke, R.; Rieger, J. Langmuir 2008, 24, 1617.

(15) Roth, S. V.; Burghammer, M.; Riekel, C.; Müller-Buschbaum, P.; Diethert,

A.; Panagiotou, P.; Walter, H. Appl. Phys. Lett. 2003, 82, 1935.

(16) Shinar, J.; Shinar, R.; Zhou, Z. Appl. Surf. Sci. 2007, 254, 749. application. Solution casting of individual droplets allows one to study structures and morphologies present after deposition on solid wetting substrates. This offers a deeper understanding of the structures induced by the solvent evaporation and its implications for coating, which is of great interest for inkjet printing. ${ }^{17}$ In an individual droplet, a multitude of structures is formed, depending on the wetting behavior of the substrate, for example, the rim and a wetting region. The wetting region might extend far from the droplet rim and could be used for installing large scale arrays of ordered colloidal and nanoparticles with respect to high-throughput applications such as surface enhanced Raman scattering (SERS), ${ }^{18}$ colorimetric detection of biopolymers, ${ }^{19}$ or sensing. ${ }^{20,21}$

Nanostructuring during colloidal solution casting is a complex, nonequilibrium process. The self-assembly involves the interplay of several flow and time-dependent mechanisms. It involves rheology, phase changes, and nonequilibrium thermodynamics. ${ }^{22,23}$ In detail, the nanostructuring takes places at the triple phase contact line air-colloidal solution-substrate. ${ }^{11}$ The process can be described as follows: During solvent evaporation, the droplet cools at the interface and the concentration of the colloidal particles increases. The evaporation rate is nonuniform and increased near the contact line. This leads to a convective flow and material transport toward the contact line ${ }^{24}$ or in the inverse direction, depending on the ratio of the surface tensions of solute and solvent. ${ }^{25}$ Compositional $^{22}$ and temperature ${ }^{26}$ driven Maragoni flows pull fluid toward the

(17) Kim, D.; Jeong, S.; Park, B. K.; Moona, J. Appl. Phys. Lett. 2006, 89, 264101.

(18) Lecomte, S.; Matejka, P.; Baron, M. H. Langmuir 1998, 14, 4373.

(19) Sarangi, S. N.; Goswani, K.; Sahu, S. N. Biosens. Bioelectron. 2007, 22, 3086.

(20) Strick, T. R.; Allemand, J.-F.; Bensimon, D.; Bensimon, A.; Croquette, V. Science 1996, 271, 1835.

(21) Wolkenhauer, M.; Bumbu, G.-G.; Cheng, Y.; Roth, S. V.; Gutmann, J. S. Appl. Phys. Lett. 2006, 89, 054101.

(22) Haw, M. D.; Gillie, M.; Poon, W. C. K. Langmuir 2002, 18, 1626.

(23) Müller-Buschbaum, P.; Bauer, E.; Pfister, S.; Roth, S. V.; Burghammer,

M.; Riekel, C.; David, C.; Thiele, U. Europhys. Lett. 2006, 73, 35.

(24) Gonuguntla, M.; Sharma, A. Langmuir 2004, 20, 3456

(25) Poulard, C.; Damman, P. Europhys. Lett. 2007, 80, 64001.

(26) Nguyen, V. X.; Stebe, K. J. Phys. Rev. Lett. 2002, 88, 164501. 
a)

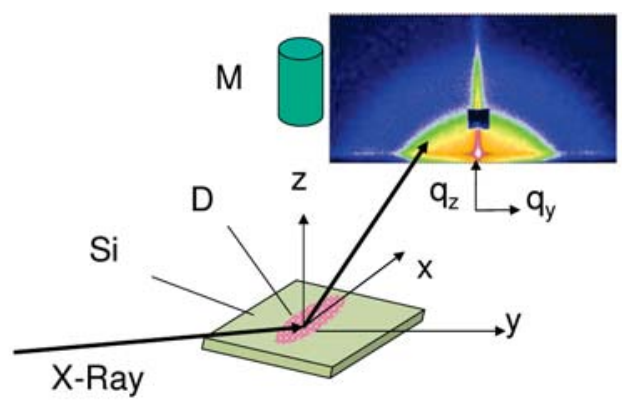

b)



Figure 1. (a) Sketch of the scattering geometry. M denotes an optical microscope, $x / y / z$ a real space coordinate system, and $q_{y}$ and $q_{z}$ denote the scattering wavevector components parallel and vertical to the sample surface. $\mathrm{D}$ denotes the dried droplet, and $\mathrm{Si}$ the silicon wafer. (b) Optical micrograph of the deposited and dried droplet (D). Exemplary nGISAXS scattering pattern from the rim $(\mathrm{R})$ and far away in the wetting region (W) are shown ( $y$ position indicated in the picture). All nGISAXS patterns are shown on a logarithmic scale. The $q$-ranges are indicated.

cooler rim and thus reinforce the instability. Furthermore, transversal contact line instabilities ${ }^{27}$ and capillary forces ${ }^{28}$ have to be taken into account. Since multiple length scales, ${ }^{29}$ from the individual nanoparticle size to micrometer or millimeter sized domains in the rim and wetting region, are involved, investigation methods featuring high spatial resolution ${ }^{11}$ in combination with high-throughput screening have to be applied. The resulting large amount of data strongly favors a new conceptional approach, namely, the use of adequate screening and classification algorithms. ${ }^{30-32}$

A dried droplet is a two-dimensional gradient and shows regions of different structures. ${ }^{17}$ Determining these structures is essential for future technological applications, as different structures are realized simultaneously. ${ }^{9}$ For structural investigations in thin films, atomic force microscopy and grazing incidence scattering methods have been applied. ${ }^{23}$ In order to investigate the different regions, we employed a new combinatorial method, namely, nanobeam grazing incidence small-angle $X$-ray scattering (nGISAXS). ${ }^{11}$ The experiment was performed at the beamline ID13/ESRF (distance sample-to-detector $D_{\mathrm{SD}}=0.802 \mathrm{~m}$, wavelength $\lambda=0.0976 \mathrm{~nm}$, and incidence angle $\alpha_{\mathrm{i}}=0.543^{\circ}$ ). The beam was focused to a size of $300 \mathrm{~nm}$, leading to a footprint on the sample of $0.3 \times 32 \mu \mathrm{m}^{2}$. The scattering geometry is sketched in Figure 1a. The sample was prepared as follows: A droplet

(27) Deegan, R. D. Phys. Rev. E 2000, 61, 475.

(28) Denkov, N. D.; Velev, O. D.; Kralchevsky, P. A.; Ivanov, I. B.; Yoshimura, H.; Nagayama, K. Nature 1993, 361, 26.

(29) Roth, S. V.; Müller-Buschbaum, P.; Timmann, A.; Perlich, J.; Gehrke, R. J. Appl. Crystallogr. 2007, 40, s346.

(30) Canty, M. J. Comput. Geosci. 2009, 35, 1280.

(31) Wroblewski, T.; Wild, E.; Poeste, T.; Pyzalla, A. J. Mater. Sci. Lett. 2000, 19,975 .

(32) Duda, T.; Canty, M. J. Int. J. Remote Sensing 2002, 23, 2193.

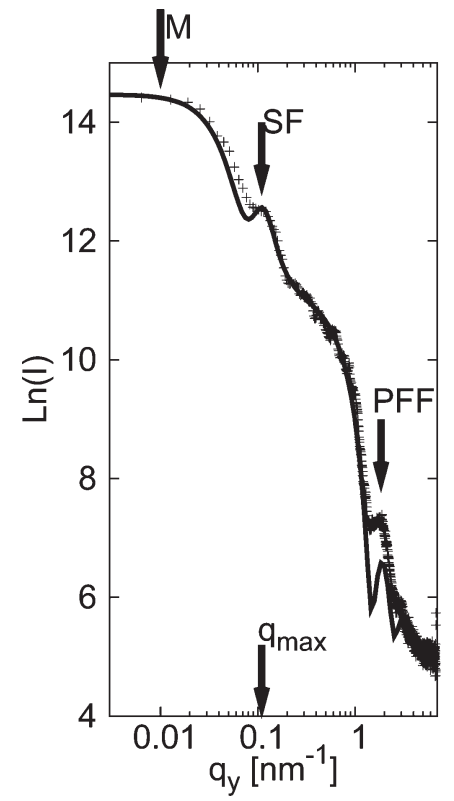

Figure 2. Exemplary out-of-plane cut taken in region D of Figure $1 \mathrm{~b}$ for $y=140 \mu \mathrm{m}$ and resulting fit of the model function (eq 2). The resolution broadened central maximum $(\mathrm{M})$, the structure factor (SF) with its maximum at $q_{\max }$, and the particle form factor (PFF) are highlighted.

(volume of $25 \mu \mathrm{L}$, diameter of $3.6 \mathrm{~mm}, 5 \mathrm{~nm}$ spherical gold nanoparticles in aqueous solution with a concentration of $5 \times$ $10^{13}$ particles $/ \mathrm{mL}$ ) was deposited on acid cleaned silicon and dried under ambient conditions $\left(T=22{ }^{\circ} \mathrm{C}, \sim 30 \%\right.$ humidity). Immediately after deposition, the droplet wetted the Si surface. The dried droplet was subsequently scanned using nGISAXS with a step size of $\Delta y=2 \mu \mathrm{m}$, thereby allowing one to distinguish different structural regions present in the dried droplet caused by the nonlinear processes during drying. Figure $1 \mathrm{~b}$ shows an optical micrograph of the dried droplet and selected scattering patterns, one with a corresponding enlargement for better displaying of the nanostructure peaks. The nGISAXS pattern obtained at $y=$ $408 \mu \mathrm{m}$ corresponds to the optically visible outer rim of the droplet. The selected patterns show significant changes from a close-packed colloidal film to a large-scale nanostructure (seen at $y=1054 \mu \mathrm{m}$ ), occurring beyond the rim. We note that in principle the origin of $y$ can be chosen arbitrarily. However, as we focus on the region around the rim and outward of the rim (visible directly after deposition), we arbitrarily chose as origin for $y$ a point at a distance of $400 \mu \mathrm{m}$ away from the rim inside the droplet. We scanned $1000 \mu \mathrm{m}$ outside from the rim to investigate the wetting region. At further distance, the signal-to-noise ratio becomes too weak.

For the first time, the analysis of GISAXS data was done by applying classification methods ${ }^{33}$ combined with manual analysis. To prepare data analysis, for each of the 700 nGISAXS patterns, the intensity distribution along the scattering vector component $q_{y}$ parallel to the sample surface was extracted. This so-called out-of-plane cut (abbreviated as oop ${ }^{7}$ ) was performed at the Yoneda peak of Si; see Figure 1a for illustration. The data analysis proceeded in two steps. First, all patterns were evaluated by unsupervised classification (USC). This allowed distinct features inside the given data set to be revealed and served as basis for a detailed manual analysis, which was made on a data subset.

(33) Canty, M. J. Image Analysis, Classification and Change Detection in Remote Sensing, with Algorithms for ENVI/IDL; CRC Press: New York, 2007. 
Finally, supervised classification ${ }^{33}$ was applied to determine the most prominent in-plane distances $\xi$ for the entire data set. $\xi$ denotes the distance of domains of nanospheres, as sketched in Figure 3.

In USC, the data set is divided into classes based on a certain similarity measure. To start with, we have chosen randomly selected oop-scans $I\left(q_{y}\right)$ to initialize the iterative clustering. Assuming six potential classes $j=1 \ldots 6$, we used as an initial approach the Euclidean distance as a similarity measure to discriminate the logarithm of the intensity $\ln \left(I\left(q_{y}\right)\right)$ by

$$
\sigma_{j}^{2}=\sum_{q_{y}}\left[\ln \left(I_{y}\left(q_{y}\right)\right)-\ln \left(I_{j}\left(q_{y}\right)\right)\right]^{2}
$$

with $I_{y}\left(q_{y}\right),-0.3 \mathrm{~nm}^{-1} \leq q_{y} \leq 2.9 \mathrm{~nm}^{-1}$, denoting the oop-scan at position $y$ (a so-called k-means clustering). By this iterative approach, at each iteration step, the oop-scans are assigned to the class having the largest similarity, that is, smallest $\sigma_{j}$. Then, new mean class representations (mean oop-scans) are calculated. The iteration is repeated until none of the mean oop-scans change significantly. We obtained the grouping shown in Figure 3a. It suggests multiple regions, separating areas along the scan line ranging from inside the droplet (left side of image) to the wetting region (right side of image). The first two regions $(0-410 \mu \mathrm{m}$, $412-650 \mu \mathrm{m})$ appear well separated, whereas the right half $(652-1400 \mu \mathrm{m})$ is banded. Assuming six classes shows as a reasonable approach; testing even more classes leads to the same oscillating features. This number overestimates our expectation and allows for later manual reduction of similar classes. Based on the division into classes obtained from USC, we chose a data subset consisting of each eighth oop-scan for manual quantitative analysis to extract the structure and morphology of the domains. Following the procedure from Beaucage, ${ }^{34}$ we used a factorized, analytical model to extract the most-prominent in-plane length scale $\xi$ as well as the particle radii using

$$
I\left(q_{y}\right)=M\left(q_{y}\right)+\operatorname{SF}\left(q_{y}\right)+\operatorname{PFF}\left(q_{y}\right)
$$

where $M\left(q_{y}\right)$ denotes the Gaussian resolution function for the beam, and $\operatorname{SF}\left(q_{y}\right)$ is an experimental Lorentzian structure factor

$$
\mathrm{SF}\left(q_{y}\right) \propto \frac{1}{1+4\left(\left(q_{y}-q_{\max }\right) / \omega\right)^{2}}
$$

with $\omega$ being its width. $\operatorname{PFF}\left(q_{y}\right)$ is the particle form factor for spheres with a nominal diameter of $5 \mathrm{~nm}$. An exemplary out-ofplane cut with the resulting fit of the model is shown in Figure 2. We relate $q_{\max }$ to $\xi$ via $\xi=2 \pi / q_{\max }$. Obtained $\xi$ values from this modeling are shown in Figure $3 \mathrm{~b}$ by tilted square symbols. Comparing the clustering result found by unsupervised classification in Figure 3a to the result from manual analysis in Figure 3b, one can see a correspondence. Even though USC uses no underlying physical model, one observes a separation into regions $\mathrm{R} 1$, R2, R3 with different $\xi$ values and a very good agreement with the manual analysis. The clustering leads to a unique blue cluster in Figure $3 \mathrm{a}$, which would correspond to a constant $\xi$ value. This is in contrast to the change in $\xi$ around the rim, found by manual analysis. This strong change in $\xi$ around $y=400 \mu \mathrm{m}$ is not sufficiently represented. To relate a physical value to each group, the oop-scans in Figure 3d justify to choose the most-prominent in-plane length $\xi$. To determine an adequate representation of the

(34) Beaucage, G. J. Appl. Crystallogr. 1995, 28, 717.
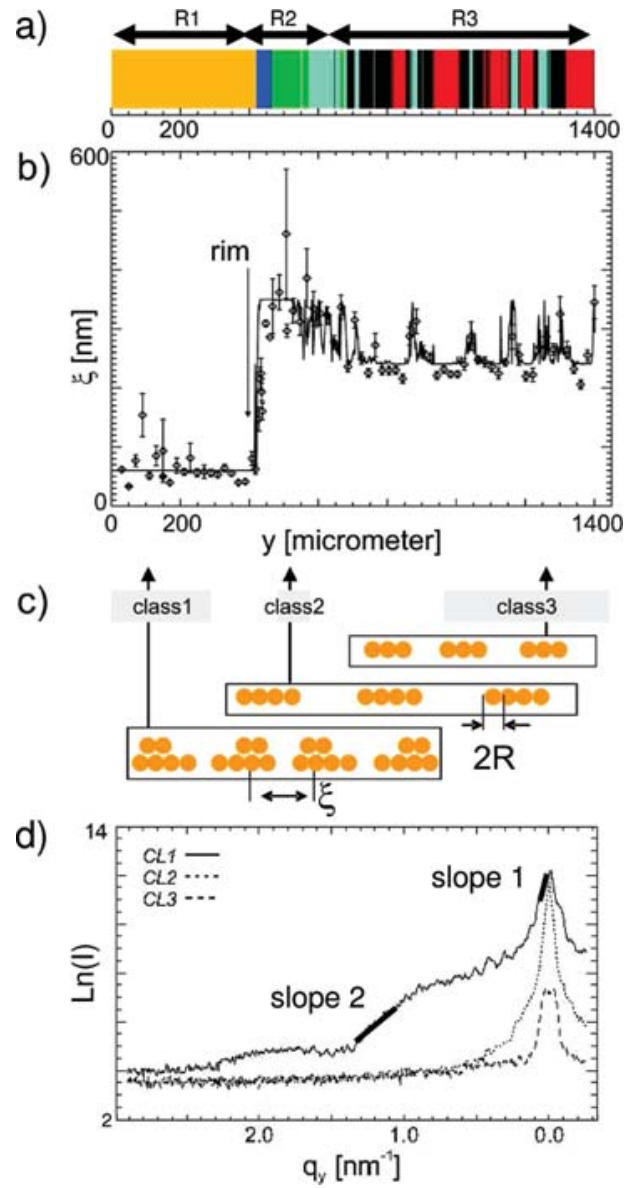

Figure 3. (a) Results from unsupervised classification for six classes (indicated by different colors): the transition from the droplet $(\mathrm{R} 1, \mathrm{R} 2)$ to an oscillating regime (R3) can be seen. (b) Comparison of most prominent in-plane lengths $\xi$ obtained by manual analysis (symbols) and by linear combination (solid line) of supervised classification. The optical rim of the droplet is indicated. (c) Model for the domain structure, dominating in R1, R2,R3: $\xi$ nanoparticle cluster distance, $2 R$ nanoparticle diameter. Gray bars denote the regions of reference classes. (d) Selected oopscans ( $y=200,460,1360 \mu \mathrm{m}$ from top to bottom) and their class representation CL1,2,3 used for maximum likelihood classification. Thick black lines illustrate slopes 1,2.

most prominent in-plane distances, we applied supervised maximum likelihood classification. We used the results obtained from the model based manual analysis to establish three classes representing three different $\xi$ values. Manually analyzed data belonging to positions lower than $300 \mu \mathrm{m}$ were defined as "class 1 " representing an average $\xi$ of $60 \mathrm{~nm}$, data belonging to positions in between 480 and $600 \mu \mathrm{m}$ were considered "class 2 " having $\xi$ of $350 \mathrm{~nm}$, and data for $y \geq 1000 \mu \mathrm{m}$ were allocated to "class 3 "representing $\xi$ of $240 \mathrm{~nm}$. Selected oop-scans at $y=200,460$, and $1360 \mu \mathrm{m}$ are illustrated in Figure 3d. Note that the regions showing the strong slope (strong increase of $\xi$ around $400 \mu \mathrm{m}$ ) as well as the region between $600 \mu \mathrm{m}<y<1000 \mu \mathrm{m}$, showing a moderate decay, have not been considered. The horizontal scale is presented linearly, as we thereby are able to emphasize the different slopes, which would be hardly visible in a logarithmic scale.

Class representations have been broken down to two dimensions; that is, the slopes for $0.02 \mathrm{~nm}^{-1}<q_{y}<0.06 \mathrm{~nm}^{-1}$ and $1.03 \mathrm{~nm}^{-1}<q_{y}<1.33 \mathrm{~nm}^{-1}$ were used as input feature. These slopes 1 and 2 allow for defining training sets in terms of arithmetic mean slopes and covariances derived from the manually analyzed data sets. For any remaining oop-scan, that is, any 

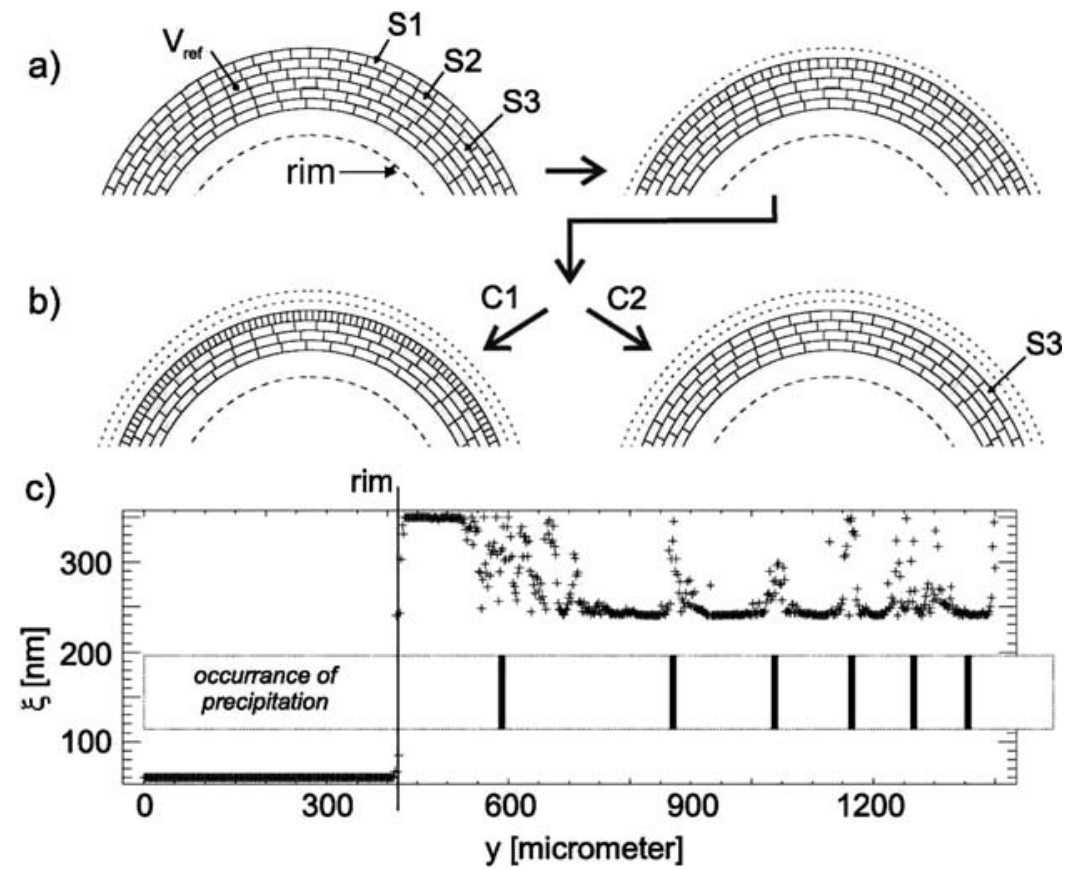

Figure 4. (a) Sketch of the model for the drying droplet. $V_{\text {ref }}$ represents a volume filled with $N$ colloidal particles. S1, S2, S3 denote the three outermost shells for illustration. (b) Illustration of the stick-slip behavior and the occurrence of precipitation. C1 shows the case of further retraction and dragging, and $\mathrm{C} 2$ the case of precipitation when a critical particle level is reached. Here, we observe an increase in $\xi$. (c) Comparison between the positions of increased $\xi$ deduced from the nGISAXS and the prediction of precipation occurrence from the model (inset).

oop-cut not used for training sets, we determined its likelihood $P_{i=1,2,3}(y)^{33}$ for belonging to one of the three classes. The $a$ priori probability was assumed to be $1 / 3$ for each class CL1,2,3. Training sets were extracted from the positions indicated by the gray bars in Figure 3c, where also manual analysis was performed. This corresponds to approximately $10 \%$ of the total number of oop-scans. The regions are motivated by the fact that the nanoparticles aggregate and regions of different $\xi$ are present in a dried solution cast gradient films ${ }^{29}$ and inside solution cast droplets, as found by confocal microscopy. ${ }^{17}$

In Figure 3d, we show three typical oop-scans with corresponding slopes, which belong to the class representations of CL1,2,3. We note that CL2 and CL3 are mainly distinguished by slope 1, which is related to the structure factor maximum in the relevant $q_{y}$-range. It can be regarded as a fingerprint due to a tail of the maximum. Slope 2 originates in the pure form factor of the spherical particles and their density. This provides the basis for a phenomenological analysis of the data, relating $\xi$ and the two slopes. The found oscillating behavior, deduced by relating $\xi$ and slope 1,2 , is corroborated by a simple physical model, which is explained below. From supervised classification, for each oop-scan at position $y$ along the profile, we thus obtain its likelihoods $P_{i}(y)$ to belong to a given class reference. Finally, for a single oop-scan at position $y$, we determined the likelihood based $\xi_{\mathrm{L}}(y)$ by linear combination of its likelihood $P_{i}(y)$ to each CLi $\left.i=1,2,3\right)$ and its corresponding (user defined reference) scale $\xi_{i}, i=1,2,3$ :

$$
\xi_{\mathrm{L}}(y)=P_{1}(y) \xi_{1}+P_{2}(y) \xi_{2}+P_{3}(y) \xi_{3}
$$

The result is shown as a solid line in Figure 3b. One can interpret $P_{i}(y)$ as a relative weight for a certain structure to be present at the scan position $y$. Then, eq 4 gives the mean $\xi$ values of these (three) underlying basic structures.

The supervised classification in combination with eq 4 confirms a constant $\xi$ for regions 1 and 2 . In addition, we furthermore note three striking features. First, the strong increase between region 1 and 2 for $\xi$ is reproduced by our approach. Second, the crossover between region 2 and 3 is reproduced as well. Third, we note a roughly periodic behavior of $\xi$ in region 3 . This periodic behavior corresponds to the banded region in Figure 3a. Such periodic oscillations could be expected and are well-known from nonequilibrium kinetics of solvent evaporation in droplets of colloidal or polymeric solutions. ${ }^{24,27,35}$ However, this seems to be new for the wetting region and could open new possibilities for exploiting such structures for thin film applications. In detail, we interpret these oscillations as domain formation of colloidal particles with different domain distance (see Figure 3c). One has to note that none of these three findings has been used as input in our approach. We calculated a mean relative deviation in $\xi$ of $33 \%$ between manual analysis and supervised classification. The estimate is based on an independent manual analyzed subset not used for the training data.

To elucidate the mechanism leading to the periodicity of the drying kinetics of the wetted film, we propose a simplified numerical stick-slip model based on Adachi et al. and Govor et al. ${ }^{35,36}$ Our approach is represented by the flow-diagram-like sketch in Figure 4. Initially, after deposition of the colloidal solution, the nanoparticles are homogeneously distributed throughout the volume of the droplet. After drying, the rim marks the visible boundary of the droplet with the optically nonvisible wetting area being outside this rim (see Figure 4a). The droplet volume outside the rim can be divided into equidistant shells. Each shell is divided into small volume elements, $V_{\text {ref }}$, filled with $N$ colloidal particles. In the given illustration (Figure $4 \mathrm{a}$ ), we sketch this model with the three outermost shells S1, S2, S3. Upon drying, the triple-phase boundary line (TPBL) at S1 retracts and drags the colloids into the next inner shell S2, increasing the particle number in this shell. If the

(35) Adachi, E.; Dimitrov, A. S.; Nagayama, K. Langmuir 1995, 11, 1057. (36) Govor, L.; Reiter, G.; Bauer, G.; Parisi, J. Appl. Phys. Lett. 2004, 84, 4774. 
particle number exceeds a critical level, the colloidal particles precipitate. Then, the retraction of the TPBL continues starting at the next shell S3 (see Figure $4 b$, case C2). With the TPBL retracting further, the process of dragging and precipitation upon a critical particle level is repeated. In the other case, $N<N_{\text {crit }}$, all particles are dragged to the next shell $\mathrm{S} 3$ (Figure $4 \mathrm{~b}$, case $\mathrm{C} 1$ ). This model leads to the distance-dependent oscillations observed in the wetting region (see Figure 4c). The spacing of the maxima, in our model indicated as singularities of the precipitation, for $\xi$ is in good agreement with the data. Maxima resulting from the model occur due to precipitation of particles; their position corresponds to regions where an increased in-plane length was found. The nanoparticles agglomerate and thus form domains. A larger amount of nanoparticles present in such a region consequently leads to the formation of larger domains and larger distances between them. Finally, we would like to note that the rim cannot be explained by

(37) Duda, R. O.; Hart, P. E.; Stork, D. G. Pattern Classification, 2nd ed.; Wiley: New York, 2001. the model, even though the majority of material is precipitated here. The rim is out of the reach of our model.

In summary, we have applied a new path for high-throughput screening and data analysis to identify patterning mechanisms in the wetting region in the vicinity of the rim of a colloidal solution droplet. Our approach combines advanced X-ray scattering and combinatorial methods and exploits analysis via classification methods used, for example, in remote sensing. Classification algorithms themselves have their origin in statistics, pattern recognition, and machine learning. ${ }^{37}$ The multiscale patterning covers a large area and changes periodically. A simple numerical model is used to explain the occurrence of these oscillations. These features render this patterning useful to create two-dimensional gradient structures for fluidic or photonic applications, for example, gratings.

Acknowledgment. This work was financially supported by the DFG Schwerpunktprogramm SPP 1164 "Nano- and Microfluidics" (Mu1487/2). 